\title{
Operator norms of Gauß-Weierstraß operators and their left quasi interpolants
}

\author{
Ulrich Abel
}

\begin{abstract}
The paper deals with the Gauß-Weierstraß operators $W_{n}$ and their left quasi interpolants $W_{n}^{[r]}$. The quasi interpolants were defined by Paul Sablonnière in 2014. Recently, their asymptotic behaviour was studied by Octavian Agratini, Radu Păltănea and the author by presenting complete asymptotic expansions. In this paper we derive estimates for the operator norms of $W_{n}$ and $W_{n}^{[r]}$ when acting on various function spaces.
\end{abstract}

Mathematics Subject Classification (2010): 41A36, 41A45, 47A30.

Keywords: Approximation by positive operators, operator norm.

\section{Introduction}

For $1 \leq p \leq+\infty$ and $c>0$, let $L_{c}^{p}(\mathbb{R})$ denote the space of all locally integrable functions $f: \mathbb{R} \rightarrow \mathbb{R}$, such that the weighted norm $\left\|f w_{c}\right\|_{L^{p}(\mathbb{R})}$

$$
\begin{aligned}
\|f\|_{L_{c}^{p}(\mathbb{R})}:= & \left(\int_{-\infty}^{\infty}|f(t)|^{p} w_{c}(t) d t\right)^{1 / p} \quad(1 \leq p<+\infty), \\
\|f\|_{L_{c}^{\infty}(\mathbb{R})}:= & \quad \operatorname{essips}_{t \in \mathbb{R}}|f(t)| w_{c}(t) \quad(p=+\infty)
\end{aligned}
$$

is finite, where the weight function $w_{c}$ is given by

$$
w_{c}(t):=e^{-c t^{2}} \text {. }
$$

In the particular case $c=0$, we obtain the ordinary spaces $L_{0}^{p}(\mathbb{R})=L^{p}(\mathbb{R})$ and $L_{0}^{\infty}(\mathbb{R})=L^{\infty}(\mathbb{R})$, respectively. 
The $n$th Gauß-Weierstraß convolution operator $W_{n}$ (see, e.g., [7, Section 5.2.9]) is defined by

$$
\left(W_{n} f\right)(x)=\sqrt{\frac{n}{\pi}} \int_{-\infty}^{\infty} e^{-n(t-x)^{2}} f(t) d t .
$$

Note that the integral on the right-hand side exists, for $f \in L_{c}^{p}(\mathbb{R})$, provided that $n>c$. We have convergence $\lim _{n \rightarrow \infty}\left(W_{n} f\right)(x)=f(x)$ in each continuity point $x \in \mathbb{R}$ of $f \in L_{c}^{p}(\mathbb{R})$. The operator $W_{n}$ played a key role in the original proof of the Weierstraß approximation theorem. The properties of $W_{n}$ have been studied by many authors (we refer to [8] for details). What regards the local rate of convergence as $n$ tends to infinity the sequence $\left(W_{n}\right)$ satisfies the Voronovskaja-type formula

$$
\lim _{n \rightarrow \infty} n\left(\left(W_{n} f\right)(x)-f(x)\right)=\frac{1}{4} f^{\prime \prime}(x),
$$

provided that the derivative $f^{\prime \prime}(x)$ exists. For more smooth functions the operators $W_{n}$ possess the complete asymptotic expansion

$$
\left(W_{n} f\right)(x) \sim f(x)+\sum_{k=1}^{\infty} \frac{1}{4^{k} k ! n^{k}} f^{(2 k)}(x) \quad(n \rightarrow \infty) .
$$

This formula follows from [4, Theorem 5.1] where it was proved for a more general sequence of operators introduced by Altomare and Milella [6, Eq. (2.5)]. Eq. (1.2) is valid also with respect to simultaneous approximation [2, Proposition 3.4.] where it turns out that the asymptotic expansion can be differentiated term-by-term. In particular, for $m=0,1,2, \ldots$, we have

$$
\lim _{n \rightarrow \infty} n\left(\left(W_{n} f\right)^{(m)}(x)-f^{(m)}(x)\right)=\frac{1}{4} f^{(m+2)}(x) .
$$

Eq. (1.2) was rediscovered, for polynomial functions, by Sablonnière [11, Theorem 1] in 2014. With this recent paper he renewed the interest in Gauß-Weierstraß operators. Sablonnière defined left and right quasi-interpolants $W_{n}^{[r]}$ and $W_{n}^{(r)}$, resp., of $W_{n}$, presented their explicit integral representations and derived a plenty of nice properties. In particular, Sablonnière [11, Theorem 5] expressed the operator norm of $W_{n}^{[r]}$ with respect to the uniform norm in terms of a certain integral which cannot be exactly evaluated. He proved that $r+\sqrt{2}$ is an upper bound on this operator norm [11, Theorem 6].

In this paper we considerably improve the upper bound. Furthermore, we study the operator norms of $W_{n}$ and $W_{n}^{[r]}$ when acting on various function spaces.

\section{The left quasi interpolants}

The Gauß-Weierstraß operators possess the representation

$$
W_{n}=\sum_{k \geq 0} \frac{1}{4^{k} k ! n^{k}} D^{2 k}
$$


as a differential operator on the space of algebraic polynomials [11, Theorem 1]. Here $D$ denotes the differentiation operator. The inverse operator $[11$, Theorem 1$]$ is given by

$$
V_{n}=\sum_{k \geq 0} \frac{(-1)^{k}}{4^{k} k ! n^{k}} D^{2 k}
$$

Composition of the the partial sums $V_{n}^{[r]}$ of order $r$ and $W_{n}$ defines the left quasi interpolants

$$
W_{n}^{[r]}:=V_{n}^{[r]} \circ W_{n}=\sum_{k=0}^{r} \frac{(-1)^{k}}{4^{k} k ! n^{k}} D^{2 k} W_{n} .
$$

[11, Subsection 4.1].

By [11, Theorem 3] (where $\tilde{H}_{2 r}(x-t)$ correctly reads $\tilde{H}_{2 r}(\sqrt{n}(x-t))$ in the first representation), the left quasi-interpolants $W_{n}^{[r]}$ of the Gauß-Weierstraß operators possess the integral representation

$$
\left(W_{n}^{[r]} f\right)(x)=\sqrt{\frac{n}{\pi}} \int_{-\infty}^{\infty} \tilde{H}_{2 r}(\sqrt{n}(x-t)) e^{-n(t-x)^{2}} f(t) d t .
$$

The polynomials $\tilde{H}_{2 r}$ are defined by

$$
\tilde{H}_{2 r}(x)=\sum_{k=0}^{r} \frac{(-1)^{k}}{4^{k} k !} H_{2 k}(x),
$$

where $H_{k}$ denote the Hermite polynomials [11, p. 38]. Sablonnière proved the explicit representation [11, Theorem 4]

$$
\tilde{H}_{2 r}(x)=\frac{(2 r+1) !}{r !} \sum_{k=0}^{r} \frac{(-1)^{r-k}}{4^{k} k !(2 r-2 k+1) !} x^{2(r-k)} .
$$

In the next section we frequently make use of the following Lemma.

Lemma 2.1. For $r=0,1,2, \ldots$, the polynomials $\tilde{H}_{2 r}$ satisfy the relation

$$
\int_{-\infty}^{\infty}\left(\tilde{H}_{2 r}(t)\right)^{2} e^{-t^{2}} d t=\sqrt{\pi}\left(\begin{array}{c}
r+1 / 2 \\
r
\end{array}\right) .
$$

Furthermore, we have the estimate

$$
2 \sqrt{r+3 / 4}<\sqrt{\pi}\left(\begin{array}{c}
r+1 / 2 \\
r
\end{array}\right) \leq 2 \sqrt{r+1} \quad(r \geq 0)
$$

and the asymptotic relation

$$
\sqrt{\pi}\left(\begin{array}{c}
r+1 / 2 \\
r
\end{array}\right) \sim 2 \sqrt{r} \quad(r \rightarrow \infty) .
$$

Remark 2.2. In other words, we have

$$
\left\|\tilde{H}_{2 r}\right\|_{L_{1}^{2}(\mathbb{R})}=\sqrt{\sqrt{\pi}\left(\begin{array}{c}
r+1 / 2 \\
r
\end{array}\right)} .
$$


Proof of Lemma 2.1. Taking advantage of the orthogonality of the Hermite polynomials (see, e.g., [5, formula 22.2.14]) we obtain

$$
\begin{aligned}
\int_{-\infty}^{\infty} \tilde{H}_{2 r}^{2}(t) \exp \left(-t^{2}\right) d t & =\sum_{k=0}^{r} \frac{(-1)^{k}}{4^{k} k !} \sum_{j=0}^{r} \frac{(-1)^{j}}{4^{j} j !} \int_{-\infty}^{\infty} H_{2 k}(x) H_{2 j}(x) e^{-t^{2}} d t \\
& =\sum_{k=0}^{r} \frac{1}{4^{2 k}(k !)^{2}} \int_{-\infty}^{\infty} H_{2 k}^{2}(x) e^{-t^{2}} d t \\
& =\sum_{k=0}^{r} \frac{1}{4^{2 k}(k !)^{2}} \sqrt{\pi} 2^{2 k}(2 k) ! \\
& =\sqrt{\pi} \sum_{k=0}^{r} \frac{1}{4^{k}}\left(\begin{array}{c}
2 k \\
k
\end{array}\right) .
\end{aligned}
$$

Application of the well-known identity [10, formulas (1.108) and (1.109)]

$$
\sum_{k=0}^{r} \frac{1}{4^{k}}\left(\begin{array}{c}
2 k \\
k
\end{array}\right)=\left(\begin{array}{c}
r+1 / 2 \\
r
\end{array}\right)
$$

proves Eq. (2.4). The bounds from below and above are a consequence of the estimate $[12]$

$$
\sqrt{y+1 / 4}<\frac{\Gamma(y+1)}{\Gamma(y+1 / 2)} \leq \sqrt{y+1 / \pi} \quad(y \geq 0) .
$$

Using $\Gamma(3 / 2)=\sqrt{\pi} / 2$, this implies

$$
\begin{aligned}
\frac{2}{\sqrt{\pi}} \sqrt{r+1 / 2+1 / 4} & <\left(\begin{array}{c}
r+1 / 2 \\
r
\end{array}\right)=\frac{\Gamma(r+3 / 2)}{\Gamma(3 / 2) \Gamma(r+1)} \\
& \leq \frac{2}{\sqrt{\pi}} \sqrt{r+1 / 2+1 / \pi} .
\end{aligned}
$$

Application of the well-known formula [5, formula 6.1.47]) yields the asymptotic relation

$$
\sqrt{\pi}\left(\begin{array}{c}
r+1 / 2 \\
r
\end{array}\right)=\Gamma(1 / 2) \frac{\Gamma(r+3 / 2)}{\Gamma(3 / 2) \Gamma(r+1)} \sim 2 \sqrt{r}\left(1+\frac{2}{r}\right) \sim 2 \sqrt{r}
$$

as $r \rightarrow \infty$.

\section{The operator norms of $W_{n}$ and $W_{n}^{[r]}$ in the space $L^{\infty}(\mathbb{R})$}

We consider the operator norm of

$$
W_{n}^{[r]}: L^{\infty}(\mathbb{R}) \rightarrow L^{\infty}(\mathbb{R})
$$

with respect to the sup-norm on $L^{\infty}(\mathbb{R})$. Sablonnière [11, Theorem 5] gave the following result. 
Proposition 3.1. The operator norm with respect to the sup-norm on $L^{\infty}(\mathbb{R})$ is given by

$$
\left\|W_{n}^{[r]}\right\|_{\left(L^{\infty}(\mathbb{R}), L^{\infty}(\mathbb{R})\right)}=\frac{1}{\sqrt{\pi}} \int_{-\infty}^{\infty}\left|\tilde{H}_{2 r}(t)\right| e^{-t^{2}} d t .
$$

Note that the value of $\left\|W_{n}^{[r]}\right\|_{\left(L^{\infty}(\mathbb{R}), L^{\infty}(\mathbb{R})\right)}$ is independent of $n$. As in [11, Theorem 5] we put, for the sake of brevity,

$$
N_{r}:=\left\|W_{n}^{[r]}\right\|_{\left(L^{\infty}(\mathbb{R}), L^{\infty}(\mathbb{R})\right)} .
$$

Remark 3.2. In the special case $r=0$ we obtain the well-known operator norm $\left\|W_{n}\right\|_{\left(L^{\infty}(\mathbb{R}), L^{\infty}(\mathbb{R})\right)}=1$ of the Gauß-Weierstraß operator $W_{n}$, since $\tilde{H}_{0}(x)=1$.

Since the proof given in [11] is not completely correct we present a proof.

Proof of Prop. 3.1. Let $f \in L^{\infty}(\mathbb{R})$. By Eq. (2.1), we have

$$
\left(W_{n}^{[r]} f\right)(x)=\frac{1}{\sqrt{\pi}} \int_{-\infty}^{\infty} \tilde{H}_{2 r}(t) e^{-t^{2}} f\left(x-\frac{t}{\sqrt{n}}\right) d t .
$$

Hence, for all $x \in \mathbb{R}$,

$$
\left|\left(W_{n}^{[r]} f\right)(x)\right| \leq N_{r} \cdot\|f\|_{L^{\infty}(\mathbb{R})}
$$

which implies

$$
\left\|W_{n}^{[r]} f\right\|_{L^{\infty}(\mathbb{R})} \leq N_{r}
$$

The function $f_{0}(t)=\operatorname{sgn}\left(\tilde{H}_{2 r}(-\sqrt{n} t)\right)$ satisfies

$$
\left\|W_{n}^{[r]} f_{0}\right\|_{L^{\infty}(\mathbb{R})} \geq\left(W_{n}^{[r]} f_{0}\right)(0)=N_{r}=N_{r} \cdot\left\|f_{0}\right\|_{L^{\infty}(\mathbb{R})}
$$

which completes the proof.

Using the well-known estimate $\left|H_{2 r}(x)\right| \leq 2^{r} \sqrt{(2 r) !} e^{x^{2} / 2}$ (see, e.g., $[9$, Subsection 1.5.1, p. 31]), Sablonnière [11, Theorem 6] proves, for $r=0,1,2, \ldots$, the both estimates

$$
N_{r} \leq \sqrt{2} \sigma_{r}:=\sqrt{2}\left(1+\sum_{p=1}^{r} \sqrt{\prod_{j=1}^{p} \frac{2 j-1}{2 j}}\right) \leq C_{r}:=r+\sqrt{2} .
$$

We improve these upper bounds as follows.

Theorem 3.3. For $r=0,1,2, \ldots$, the operator norm $\left\|W_{n}^{[r]}\right\|_{\left(L^{\infty}(\mathbb{R}), L^{\infty}(\mathbb{R})\right)}=N_{r}$ satisfies the estimate

$$
N_{r} \leq\left(\begin{array}{c}
r+1 / 2 \\
r
\end{array}\right)^{1 / 2}=: D_{r}
$$


Lemma 2.3 implies the asymptotic relation

$$
D_{r} \sim \sqrt[4]{\frac{4 r}{\pi}} \quad(r \rightarrow \infty)
$$

and the following estimate.

Corollary 3.4. For $r=0,1,2, \ldots$, the operator norm $\left\|W_{n}^{[r]}\right\|_{\left(L^{\infty}(\mathbb{R}), L^{\infty}(\mathbb{R})\right)}=N_{r}$ possesses the upper bound

$$
N_{r} \leq \sqrt[4]{4 \frac{r+1}{\pi}}
$$

The next table shows some numerical values of $N_{r}$ up to $r=10$, its estimates $\sqrt{2} \sigma_{r}$ and $C_{r}$ by Sablonnière, followed by the new estimate $D_{r}$ from Theorem 3.3:

\begin{tabular}{c|crcc}
$r$ & $N_{r}$ & $\sqrt{2} \sigma_{r}$ & \multicolumn{1}{c}{$C_{r}$} & $D_{r}$ \\
\hline 1 & 1.14 & 2.41 & 2.41 & 1.22 \\
2 & 1.22 & 3.28 & 3.41 & 1.37 \\
3 & 1.28 & 4.07 & 4.41 & 1.48 \\
4 & 1.33 & 4.81 & 5.41 & 1.57 \\
5 & 1.37 & 5.51 & 6.41 & 1.65 \\
6 & 1.40 & 6.18 & 7.41 & 1.71 \\
7 & 1.43 & 6.83 & 8.41 & 1.77 \\
8 & 1.45 & 7.46 & 9.41 & 1.83 \\
9 & 1.47 & 8.07 & 10.41 & 1.88 \\
10 & 1.49 & 8.66 & 11.41 & 1.92
\end{tabular}

Proof of Theorem 3.3. By Prop. 3.1, we have

$$
\left\|W_{n}^{[r]}\right\|_{\left(L^{\infty}(\mathbb{R}), L^{\infty}(\mathbb{R})\right)}=\frac{1}{\sqrt{\pi}} \int_{-\infty}^{\infty}\left|\tilde{H}_{2 r}(t)\right| \sqrt{e^{-t^{2}}} \sqrt{e^{-t^{2}}} d t .
$$

Application of the Schwarz inequality implies that

$$
N_{r} \leq \frac{1}{\sqrt{\pi}} \sqrt{\int_{-\infty}^{\infty}\left|\tilde{H}_{2 r}(t)\right|^{2} e^{-t^{2}} d t} \sqrt{\int_{-\infty}^{\infty} e^{-t^{2}} d t}=\left(\begin{array}{c}
r+1 / 2)^{1 / 2} \\
r
\end{array}\right)^{2}
$$

where the last equality follows from Eq. (2.4) of Lemma 2.1.

\section{The operator norms of $W_{n}$ and $W_{n}^{[r]}$ in weighted spaces}

\subsection{Weighted spaces}

In the following we suppose that $c>0$. Put $f_{c}=w_{-c}$, i.e.,

$$
f_{c}(t)=e^{c t^{2}} \text {. }
$$

Then, for all $n>c$,

$$
W_{n} f_{c}=\sqrt{\frac{n}{n-c}} f_{n c /(n-c)} .
$$


Note that, for $c>0$, it holds $c<n c /(n-c)$. This means that the space $L_{c}^{\infty}(\mathbb{R})$ is not invariant under the mapping $W_{n}$. The function $f_{c} \in L_{c}^{\infty}(\mathbb{R})$ satifies $\left\|f_{c}\right\|_{L_{c}^{\infty}(\mathbb{R})}=1$. However, $W_{n} f_{c} \notin L_{c}^{\infty}(\mathbb{R})$. Therefore, we consider the mapping $W_{n}: L_{c}^{\infty}(\mathbb{R}) \rightarrow$ $L_{\gamma}^{\infty}(\mathbb{R})$, for some $\gamma>c$. Note that $\lim _{n \rightarrow \infty} n c /(n-c)=c$ implies that $n c /(n-c)<\gamma$, for sufficiently large integers $n$. More precisely, we have $W_{n} f_{c} \in L_{\gamma}^{\infty}(\mathbb{R})$, for all integers $n$ satisfying

$$
n>\gamma c /(\gamma-c)
$$

In the following we consider only such values of $n$.

\subsection{The space $L_{c}^{\infty}(\mathbb{R})$ equipped with the weighted sup-Norm}

Let $c>0$. Suppose that $f \in L_{c}^{\infty}(\mathbb{R})$ and $W_{n}^{[r]} f \in L_{\gamma}^{\infty}(\mathbb{R})$, for some $\gamma>c$. As we know, this is the case if $n>\gamma c /(\gamma-c)$. For these $n$, the operator norm $\left\|W_{n}^{[r]}\right\|=\left\|W_{n}^{[r]}\right\|_{\left(L_{c}^{\infty}(\mathbb{R}), L_{\gamma}^{\infty}(\mathbb{R})\right)}$ is defined by

$$
\left\|W_{n}^{[r]}\right\|=\sup _{\substack{f \in L_{c}^{\infty}(\mathbb{R}) \\ f \neq 0}} \frac{\left\|W_{n}^{[r]} f\right\|_{L_{\gamma}^{\infty}(\mathbb{R})}}{\|f\|_{L_{c}^{\infty}(\mathbb{R})}}=\sup _{\substack{f \in L_{c}^{\infty}(\mathbb{R}) \\\|f\|_{L_{c}^{\infty}(\mathbb{R})}=1}}\left\|W_{n}^{[r]} f\right\|_{L_{\gamma}^{\infty}(\mathbb{R})} .
$$

Theorem 4.1. Let $r \in \mathbb{N}$. If $0<c<\gamma$, for all integers $n>2 \gamma c /(\gamma-c)$, the operator norm $\left\|W_{n}^{[r]}\right\|_{\left(L_{c}^{\infty}(\mathbb{R}), L_{\gamma}^{\infty}(\mathbb{R})\right)}$ satisfies the estimate

$$
\left\|W_{n}^{[r]}\right\|_{\left(L_{c}^{\infty}(\mathbb{R}), L_{\gamma}^{\infty}(\mathbb{R})\right)} \leq\left(\begin{array}{c}
r+1 / 2 \\
r
\end{array}\right)^{1 / 2}\left(\frac{n(\gamma-c)}{n(\gamma-c)-2 c \gamma}\right)^{1 / 4} .
$$

Remark 4.2. By Eq. (2.5) of Lemma 2.1, we have the upper bound

$$
\left\|W_{n}\right\|_{\left(L_{c}^{\infty}(\mathbb{R}), L_{\gamma}^{\infty}(\mathbb{R})\right)} \leq \sqrt[4]{4 \frac{r+1}{\pi}\left(\frac{n(\gamma-c)}{n(\gamma-c)-2 c \gamma}\right)} .
$$

Remark 4.3. In the special case $r=0$, we obtain the estimate

$$
\left\|W_{n}\right\|_{\left(L_{c}^{\infty}(\mathbb{R}), L_{\gamma}^{\infty}(\mathbb{R})\right)} \leq\left(\frac{n(\gamma-c)}{n(\gamma-c)-2 c \gamma}\right)^{1 / 4}
$$

for the classical Gauß-Weierstraß operators $W_{n}=W_{n}^{[0]}$.

Remark 4.4. The limit $n \rightarrow \infty$ leads for $\left\|W_{n}^{[r]}\right\|_{\left(L_{c}^{\infty}(\mathbb{R}), L_{\gamma}^{\infty}(\mathbb{R})\right)}$ to the upper bound

$$
D_{r}=\sqrt{\left(\begin{array}{c}
r+1 / 2 \\
r
\end{array}\right)} \sim \sqrt[4]{\frac{4 r}{\pi}} \quad(r \rightarrow \infty) .
$$

Proof of Theorem 4.1. Let $f \in L_{c}^{\infty}(\mathbb{R})$. By Eq. (2.1), we have

$$
\left(W_{n}^{[r]} f\right)(x)=\sqrt{\frac{n}{\pi}} \int_{-\infty}^{\infty} \tilde{H}_{2 r}(\sqrt{n}(x-t)) e^{-n(t-x)^{2}} w_{-c}(t) \cdot w_{c}(t) f(t) d t .
$$


which can be estimated by

$$
\begin{aligned}
\left|\left(W_{n}^{[r]} f\right)(x)\right| \leq & \sqrt{\frac{n}{\pi}} \int_{-\infty}^{\infty}\left|\tilde{H}_{2 r}(\sqrt{n}(x-t))\right| \exp \left(-n(t-x)^{2}\right) w_{-c}(t) d t \\
& \times \sup _{t \in \mathbb{R}}|f(t)| w_{c}(t) .
\end{aligned}
$$

A change of variable replacing $t$ with $x-t / \sqrt{n}$ yields

$$
\left|\left(W_{n}^{[r]} f\right)(x)\right| \leq \frac{1}{\sqrt{\pi}} \int_{-\infty}^{\infty}\left|\tilde{H}_{2 r}(t)\right| e^{-t^{2}+c(x-t / \sqrt{n})^{2}} d t \cdot\|f\|_{L_{c}^{\infty}(\mathbb{R})} .
$$

Hence,

$$
\begin{aligned}
\left\|W_{n}^{[r]} f\right\|_{L_{\gamma}^{\infty}(\mathbb{R})} & =\sup _{x \in \mathbb{R}}\left|\left(W_{n}^{[r]} f\right)(x)\right| e^{-\gamma x^{2}} \\
& \leq \sup _{x \in \mathbb{R}}\left(\frac{1}{\sqrt{\pi}} \int_{-\infty}^{\infty}\left|\tilde{H}_{2 r}(t)\right| e^{-t^{2}+c(x-t / \sqrt{n})^{2}} d t \cdot e^{-\gamma x^{2}}\right) \cdot\|f\|_{L_{c}^{\infty}(\mathbb{R})} .
\end{aligned}
$$

For fixed $t$, the expression $e^{c(x-t / \sqrt{n})^{2}} e^{-\gamma x^{2}}$ attains (as a function of $x$ ) its maximum at $x=-c t /(\sqrt{n}(\gamma-c))$, such that

$$
\sup _{x \in \mathbb{R}} e^{c(x-t / \sqrt{n})^{2}} e^{-\gamma x^{2}}=\exp \left(\frac{c \gamma}{n(\gamma-c)} t^{2}\right) \text {. }
$$

Consequently,

$$
\left\|W_{n}^{[r]} f\right\|_{L_{\gamma}^{\infty}(\mathbb{R})} \leq \frac{1}{\sqrt{\pi}} \int_{-\infty}^{\infty}\left|\tilde{H}_{2 r}(t)\right| \exp \left(-\left(1-\frac{c \gamma}{n(\gamma-c)}\right) t^{2}\right) d t \cdot\|f\|_{L_{c}^{\infty}(\mathbb{R})} .
$$

Hence,

$$
\left\|W_{n}^{[r]}\right\|_{\left(L_{c}^{\infty}(\mathbb{R}), L_{\gamma}^{\infty}(\mathbb{R})\right)} \leq \frac{1}{\sqrt{\pi}} \int_{-\infty}^{\infty}\left|\tilde{H}_{2 r}(t)\right| \exp \left(-\left(1-\frac{c \gamma}{n(\gamma-c)}\right) t^{2}\right) d t
$$

Application of the Schwarz inequality yields

$$
\begin{aligned}
& \int_{-\infty}^{\infty}\left|\tilde{H}_{2 r}(t)\right| \exp \left(-\left(1-\frac{c \gamma}{n(\gamma-c)}\right) t^{2}\right) d t \\
= & \int_{-\infty}^{\infty}\left|\tilde{H}_{2 r}(t)\right| \sqrt{e^{-t^{2}}} \cdot \sqrt{e^{t^{2}}} \exp \left(-\left(1-\frac{c \gamma}{n(\gamma-c)}\right) t^{2}\right) d t \\
\leq & \sqrt{\int_{-\infty}^{\infty}\left(\tilde{H}_{2 r}(t)\right)^{2} e^{-t^{2}} d t} \sqrt{\int_{-\infty}^{\infty} \exp \left(-\left(1-\frac{2 c \gamma}{n(\gamma-c)}\right) t^{2}\right) d t} \\
= & \sqrt{\sqrt{\pi}\left(\begin{array}{c}
r+1 / 2 \\
r
\end{array}\right)} \sqrt{\sqrt{\pi}\left(1-\frac{2 c \gamma}{n(\gamma-c)}\right)^{-1 / 2}} .
\end{aligned}
$$

By Lemma 2.1, and using the well-known identity $\int_{-\infty}^{\infty} \exp \left(-a t^{2}\right) d t=\sqrt{\pi / a}$, for $a>0$, we obtain

$$
\left\|W_{n}^{[r]}\right\|_{\left(L_{c}^{\infty}(\mathbb{R}), L_{\gamma}^{\infty}(\mathbb{R})\right)} \leq \sqrt{\left(\begin{array}{c}
r+1 / 2 \\
r
\end{array}\right)\left(1-\frac{2 c \gamma}{n(\gamma-c)}\right)^{-1 / 2}}
$$


which completes the proof.

\subsection{The space $L_{c}^{1}(\mathbb{R})$}

Now we consider the case $p=1$.

Theorem 4.5. Let $f \in L_{c}^{1}(\mathbb{R})$. Then, for all integers $n>2 c$ and each real $\gamma>$ $c(1-2 c / n)^{-1}$, it holds

$$
\left\|W_{n}^{[r]}\right\|_{\left(L_{c}^{1}(\mathbb{R}), L_{\gamma}^{\infty}(\mathbb{R})\right)} \leq \tau_{r} \sqrt{\frac{n}{\pi}}
$$

and

where

$$
\left\|W_{n}^{[r]}\right\|_{\left(L_{c}^{1}(\mathbb{R}), L_{\gamma}^{1}(\mathbb{R})\right)} \leq \tau_{r} \sqrt{\frac{n-2 c}{(1-2 c / n) \gamma-c}}
$$

$$
\tau_{r}:=\sum_{j=0}^{r} \frac{1}{2^{j}}\left(\begin{array}{c}
2 j \\
j
\end{array}\right)^{1 / 2} .
$$

Proof. Let $n>2 c$. For $f \in L_{c}^{1}(\mathbb{R})$, we obtain

$$
\left|\left(W_{n}^{[r]} f\right)(x)\right| \leq K_{n, c}(x)\|f\|_{L_{c}^{1}(\mathbb{R})}
$$

with

$$
\begin{aligned}
K_{n, c}(x) & =\sqrt{\frac{n}{\pi}} \sup _{t \in \mathbb{R}}\left|\tilde{H}_{2 r}(\sqrt{n}(x-t))\right| \exp \left(-n(t-x)^{2}\right) w_{-c}(t) \\
& \leq \sqrt{\frac{n}{\pi}} \sup _{u \in \mathbb{R}}\left|\tilde{H}_{2 r}(u)\right| e^{-u^{2} / 2} \cdot \exp \left(\sup _{t \in \mathbb{R}}\left(c t^{2}-\frac{n}{2}(t-x)^{2}\right)\right) .
\end{aligned}
$$

The well-known estimate $\left|H_{2 r}(x)\right| \leq 2^{r} \sqrt{(2 r) !} e^{x^{2} / 2}$ (see, e.g., [9, Subsection 1.5.1, p. 31]) implies the estimate

$$
\left|\tilde{H}_{2 r}(u)\right| e^{-u^{2} / 2} \leq \sum_{j=0}^{r} \frac{1}{2^{j}}\left(\begin{array}{c}
2 j \\
j
\end{array}\right)^{1 / 2}=\tau_{r}
$$

which was already remarked by Sablonnière [11, Page 42]. Elementary calculus shows that $\left(c t^{2}-\frac{n}{2}(t-x)^{2}\right)$ attains its maximum at $t=x(1-2 c / n)^{-1}$. Therefore,

$$
K_{n, c}(x) \leq \sqrt{\frac{n}{\pi}} \tau_{r} \exp \left(\frac{c x^{2}}{1-2 c / n}\right)
$$

Hence, for $n>2 c$,

$$
\left\|W_{n}^{[r]}\right\|_{\left(L_{c}^{1}(\mathbb{R}), L_{\gamma}^{\infty}(\mathbb{R})\right)} \leq \sqrt{\frac{n}{\pi}} \tau_{r} \sup _{x \in \mathbb{R}} \exp \left(-\left(\gamma-\frac{c}{1-2 c / n}\right) x^{2}\right)=\sqrt{\frac{n}{\pi}} \tau_{r}
$$

and

$$
\left\|W_{n}^{[r]}\right\|_{\left(L_{c}^{1}(\mathbb{R}), L_{\gamma}^{1}(\mathbb{R})\right)} \leq \sqrt{\frac{n}{\pi}} \tau_{r} \int_{-\infty}^{\infty} \exp \left(-\left(\gamma-\frac{c}{1-2 c / n}\right) x^{2}\right) d x
$$

which implies the assertions. 


\subsection{The spaces $L_{c}^{p}(\mathbb{R})$ with $p>1$}

Now we turn to the case $p>1$. As usual let $q$ denote the conjugate number of $p$ satisfying $1 / p+1 / q=1$.

Theorem 4.6. Let $p>1, q=p /(p-1)$, and $f \in L_{c}^{p}(\mathbb{R})$. Then, for $\gamma>c /(p-1)$ and sufficiently large integers $n$, it holds

$$
\begin{aligned}
\left\|W_{n}^{[r]}\right\|_{\left(L_{c}^{p}(\mathbb{R}), L_{\gamma}^{q}(\mathbb{R})\right)} \leq & \left(\frac{n}{\pi}\right)^{\frac{1}{2 p}}\left(\frac{p}{p \gamma-q c}\right)^{\frac{1}{2 q}} \\
& \times\left(\int_{-\infty}^{\infty}\left|\tilde{H}_{2 r}(t)\right|^{q} \exp \left(-q t^{2}\right) \exp \left(\frac{c q \gamma}{(p \gamma-q c) n} t^{2}\right) d t\right)^{1 / q} .
\end{aligned}
$$

Remark 4.7. In the special case $r=0$ (note that $\tilde{H}_{0}(t)=1$ ) one can explicitly calculate the integral

$$
\int_{-\infty}^{\infty} \exp \left(-q t^{2}\right) \exp \left(\frac{c \gamma q}{(p \gamma-c q) n} t^{2}\right) d t=\sqrt{\frac{\pi}{q-\frac{c \gamma q}{(p \gamma-c q) n}}}
$$

which tends to $\sqrt{\pi / q}$ as $n \rightarrow \infty$. Hence, for the Gauß-Weierstrass operators $W_{n}$ holds

$$
\left\|W_{n}\right\|_{\left(L_{c}^{p}(\mathbb{R}), L_{\gamma}^{q}(\mathbb{R})\right)} \leq n^{\frac{1}{2 p}} \pi^{\frac{1}{2}-\frac{1}{p}}\left(\frac{p-1}{p \gamma-c q-\frac{c \gamma}{n}}\right)^{\frac{1}{2 q}}
$$

Proof. We estimate

$$
\left(W_{n}^{[r]} f\right)(x)=\sqrt{\frac{n}{\pi}} \int_{-\infty}^{\infty} \tilde{H}_{2 r}(\sqrt{n}(x-t)) e^{-n(t-x)^{2}} w_{-c / p}(t) \cdot w_{c / p}(t) f(t) d t
$$

by Hölder's inequality (note that $\frac{1}{2}-\frac{1}{2 q}=\frac{1}{2 p}$ ):

$$
\begin{aligned}
\left|\left(W_{n}^{[r]} f\right)(x)\right| & \leq \sqrt{\frac{n}{\pi}}\left(\int_{-\infty}^{\infty}\left|\tilde{H}_{2 r}(\sqrt{n}(x-t))\right|^{q} e^{-n q(t-x)^{2}} w_{-c / p}^{q}(t) d t\right)^{1 / q} \\
& \times\left(\int_{-\infty}^{\infty} w_{c / p}^{p}(t)|f(t)|^{p} d t\right)^{1 / p} \\
& =\frac{n^{\frac{1}{2 p}}}{\sqrt{\pi}}\left(\int_{-\infty}^{\infty}\left|\tilde{H}_{2 r}(t)\right|^{q} \exp \left(-q t^{2}\right) w_{-c q / p}\left(x-\frac{t}{\sqrt{n}}\right) d t\right)^{1 / q}\|f\|_{L_{c}^{p}(\mathbb{R})} \\
& =: C(n, r, p, x) \cdot\|f\|_{L_{c}^{p}(\mathbb{R})}
\end{aligned}
$$

Then, for $\gamma>c /(p-1)$, we have

$$
\left\|W_{n}^{[r]} f\right\|_{L_{\gamma}^{q}(\mathbb{R})} \leq\|C(n, r, p, \cdot)\|_{L_{\gamma}^{q}(\mathbb{R})}\|f\|_{L_{c}^{p}(\mathbb{R})}
$$


with

$$
\begin{aligned}
& \|C(n, r, p, \cdot)\|_{L_{\gamma}^{q}(\mathbb{R})} \\
= & \frac{n^{\frac{1}{2 p}}}{\sqrt{\pi}}\left(\int_{-\infty}^{\infty}\left(\int_{-\infty}^{\infty}\left|\tilde{H}_{2 r}(t)\right|^{q} \exp \left(-q t^{2}\right) w_{-c q / p}\left(x-\frac{t}{\sqrt{n}}\right) d t\right) w_{\gamma}(x) d x\right)^{1 / q} \\
= & \frac{n^{\frac{1}{2 p}}}{\sqrt{\pi}}\left(\int_{-\infty}^{\infty}\left(\int_{-\infty}^{\infty} w_{-c q / p}\left(x-\frac{t}{\sqrt{n}}\right) w_{\gamma}(x) d x\right)\left|\tilde{H}_{2 r}(t)\right|^{q} \exp \left(-q t^{2}\right) d t\right)^{1 / q} .
\end{aligned}
$$

A short calculation yields

$$
\begin{aligned}
& \int_{-\infty}^{\infty} w_{-c q / p}\left(x-\frac{t}{\sqrt{n}}\right) \cdot w_{\gamma}(x) d x \\
= & \int_{-\infty}^{\infty} \exp \left(c \frac{q}{p}\left(x-\frac{t}{\sqrt{n}}\right)^{2}-\gamma x^{2}\right) d x \\
= & \sqrt{\frac{\pi p}{p \gamma-c q}} \exp \left(\frac{c q \gamma}{(p \gamma-q c) n} t^{2}\right) .
\end{aligned}
$$

Furthermore,

$$
\begin{aligned}
& \|C(n, r, p, \cdot)\|_{L_{\gamma}^{q}(\mathbb{R})} \\
= & \frac{n^{\frac{1}{2 p}}}{\sqrt{\pi}}\left(\sqrt{\frac{\pi p}{p \gamma-q c}} \int_{-\infty}^{\infty}\left|\tilde{H}_{2 r}(t)\right|^{q} \exp \left(-q t^{2}\right) \exp \left(\frac{c q \gamma}{(p \gamma-q c) n} t^{2}\right) d t\right)^{1 / q} .
\end{aligned}
$$

The estimate now follows by noting that $(\sqrt{\pi})^{-1+\frac{1}{q}}=\pi^{-\frac{1}{2 p}}$.

\subsection{The special case $p=q=2$}

In the special case $p=q=2$, we obtain, for $\gamma>c$ and sufficiently large integers $n$,

$$
\|C(n, r, 2, \cdot)\|_{L_{\gamma}^{2}(\mathbb{R})}=\left(\frac{n}{\pi(\gamma-c)}\right)^{\frac{1}{4}}\left(\int_{-\infty}^{\infty}\left|\tilde{H}_{2 r}(t)\right|^{2} \exp \left(-\left(2-\frac{c \gamma}{(\gamma-c) n}\right) t^{2}\right) d t\right)^{1 / 2} .
$$

Note that $\left|\tilde{H}_{2 r}(t)\right|^{2}=\tilde{H}_{2 r}^{2}(t)$. Therefore, one can explicitly evaluate the integrals for each integer value $r$.

In particular, for $r=0$, we obtain the explicit expression

$$
\begin{aligned}
\|C(n, r, 2, \cdot)\|_{L_{\gamma}^{2}(\mathbb{R})} & =\left(\frac{n}{\pi(\gamma-c)}\right)^{\frac{1}{4}}\left(\sqrt{\frac{\pi}{2-\frac{c \gamma}{(\gamma-c) n}}}\right)^{1 / 2} \\
& =\sqrt[4]{\frac{n}{2(\gamma-c)-c \gamma / n}}
\end{aligned}
$$

Hence, Theorem 4.6 reduces to the next corollary. 
Corollary 4.8. Let $f \in L_{c}^{2}(\mathbb{R})$. Then, for $\gamma>c$ and sufficiently large integers $n$, it holds

$$
\begin{aligned}
& \left\|W_{n}^{[r]}\right\|_{\left(L_{c}^{2}(\mathbb{R}), L_{\gamma}^{2}(\mathbb{R})\right)} \\
\leq & \left(\frac{n}{(\gamma-c) \pi}\right)^{\frac{1}{4}}\left(\int_{-\infty}^{\infty}\left|\tilde{H}_{2 r}(t)\right|^{2} \exp \left(-\left(2-\frac{c \gamma}{(\gamma-c) n}\right) t^{2}\right) d t\right)^{1 / 2} .
\end{aligned}
$$

In particular, for $r=0$, the operator norm of the classical Gauß-Weierstraß operators satisfies the estimate

$$
\left\|W_{n}\right\|_{\left(L_{c}^{2}(\mathbb{R}), L_{\gamma}^{2}(\mathbb{R})\right)} \leq \sqrt[4]{\frac{n}{2(\gamma-c)-c \gamma / n}} .
$$

Concluding remark. If we allow $\gamma$ to depend on $n$, we can choose $\gamma=\frac{n}{n-c} c \equiv \gamma(n)$, such that $\exp \left(-\left(2-\frac{c \gamma}{n(\gamma-c)}\right) t^{2}\right)=\exp \left(-t^{2}\right)$, then

$$
\left\|W_{n}^{[r]}\right\|_{\left(L_{c}^{2}(\mathbb{R}), L_{\gamma(n)}^{2}(\mathbb{R})\right)} \leq\left(\frac{n(n-c)}{c^{2} \pi}\right)^{\frac{1}{4}}\left\|\tilde{H}_{2 r}\right\|_{L_{1}^{2}(\mathbb{R})} .
$$

Finally, by Eq. (2.4) of Lemma 2.3,

$$
\left\|W_{n}^{[r]}\right\|_{\left(L_{c}^{2}(\mathbb{R}), L_{\gamma(n)}^{2}(\mathbb{R})\right)} \leq \sqrt[4]{\frac{n(n-c)}{c^{2}}}\left(\begin{array}{c}
r+1 / 2 \\
r
\end{array}\right)^{1 / 2} .
$$

By estimate (2.5), it follows that

$$
\left\|W_{n}^{[r]}\right\|_{\left(L_{c}^{2}(\mathbb{R}), L_{\gamma(n)}^{2}(\mathbb{R})\right)} \leq \sqrt[4]{4 \frac{n(n-c)}{c^{2} \pi}(r+1)} .
$$

Note that $\gamma(n)$ tends to $c$ (from above) as $n \rightarrow \infty$. For large values of $n$ both norms $L_{c}^{2}(\mathbb{R})$ and $L_{\gamma(n)}^{2}(\mathbb{R})$ are close together.

\section{References}

[1] Abel, U., Asymptotic expansions for Favard operators and their left quasi-interpolants, Stud. Univ. Babeş-Bolyai Math., 56(2011), 199-206.

[2] Abel, U., Agratini, O., Păltănea, R., A complete asymptotic expansion for the quasiinterpolants of Gauß-Weierstraß operators, Mediterr. J. Math., 15(2018), Online First.

[3] Abel, U., Butzer, P.L., Complete asymptotic expansion for generalized Favard operators, Constr. Approx., 35(2012), 73-88.

[4] Abel, U., Ivan, M., Simultaneous approximation by Altomare operators, Proceedings of the 6th international conference on functional analysis and approximation theory, Acquafredda di Maratea (Potenza), Italy, Sept. 24-30, 2009, Palermo: Circolo Matemàtico di Palermo, Rend. Circ. Mat. Palermo, Serie II, Suppl., 82(2010), 177-193.

[5] Abramowitz, M., Stegun, A., Handbook of Mathematical Functions, Appl. Math. Ser. 55, National Bureau of Standards, Washington D.C., 1972.

[6] Altomare, F., Milella, S., Integral-type operators on continuous function spaces on the real line, J. Approx. Theory 152(2008), 107-124. 
[7] Altomare, F., Campiti, M., Korovkin-type Approximation Theory and its Applications, de Gruyter Studies in Mathematics 17, W. de Gruyter, Berlin, New York, 1994.

[8] Butzer, P.L., Nessel, R.J., Fourier Analysis and Approximation, Birkhäuser Verlag, Basel, 1971.

[9] Gautschi, W., Orthogonal Polynomials, Oxford University Press, 2004.

[10] Gould, H.W., Combinatorial Identities, Morgantown Print \& Bind., Morgantown, WV, 1972.

[11] Sablonnière, P., Weierstrass quasi-interpolants, J. Approx. Theory, 180(2014), 32-48.

[12] Watson, G.N., A note on gamma functions, Proc. Edinb. Math. Soc., 2(11)(1958/59); Edinburgh Math. Notes, 42(1959), 7-9.

Ulrich Abel

Technische Hochschule Mittelhessen

Department Mathematik, Naturwissenschaften und Datenverarbeitung

Wilhelm-Leuschner-Straße 13, 61169 Friedberg, Germany

e-mail: Ulrich.Abel@mnd.thm.de 